\title{
Composition-Dependent Hydrogen-Bonding Motifs and Dynamics in Brønsted Acid-Base Mixtures
}

\author{
Christian Malm, Leon A. Prädel, Bogdan A. Marekha, Maksim Grechko, and Johannes Hunger* \\ Cite This: J. Phys. Chem. B 2020, 124, 7229-7238 \\ Read Online
}

ABSTRACT: In recent years the interaction of organophosphates and imines, which is at the core of Brønsted acid organocatalysis, has been established to be based on strong ionic hydrogen bonds. Yet, besides the formation of homodimers consisting of two acid molecules and heterodimers consisting of one acid and one base, also multimeric molecular aggregates are formed in solution. These multimeric aggregates consist of one base and several acid molecules. The details of the intermolecular bonding in such aggregates, however, have remained elusive. To characterize composition-
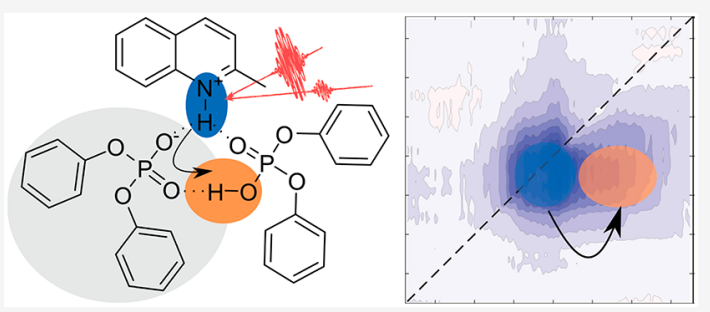
dependent bonding and bonding dynamics in these aggregates, we use linear and nonlinear infrared (IR) spectroscopy at varying molar ratios of diphenyl phosphoric acid and quinaldine. We identify the individual aggregate species, giving rise to the structured, strong, and very broad infrared absorptions, which span more than 1000 $\mathrm{cm}^{-1}$. Linear infrared spectra and density functional theory calculations of the proton transfer potential show that doubly ionic intermolecular hydrogen bonds between the acid and the base lead to absorptions which peak at $\sim 2040 \mathrm{~cm}^{-1}$. The contribution of singly ionic hydrogen bonds between an acid anion and an acid molecule is observed at higher frequencies. As common to such strong hydrogen bonds, ultrafast IR spectroscopy reveals rapid, $100 \mathrm{fs}$, dissipation of energy from the proton transfer coordinate. Yet, the full dissipation of the excess energy occurs on a $\sim 0.8-1.1$ ps time scale, which becomes longer when multimers dominate. Our results thus demonstrate the coupling and collectivity of the hydrogen bonds within these complexes, which enable efficient energy transfer.

\section{INTRODUCTION}

Aggregates formed via association of a Brønsted acid and a Brønsted base are often considered as prototypes of strong hydrogen bonds. ${ }^{1}$ The relevance of strong hydrogen bonds to, e.g., photochemical transfer cascades in biological systems, ${ }^{2,3}$ enzyme function, ${ }^{4}$ or catalysis ${ }^{5-7}$ has triggered intense interest in the understanding of their fundamental physical and chemical characteristics. $^{8-17}$ Of particular interest are such strong hydrogen bonds for fast (photo-) chemical reactions, as proton transfer along the hydrogen bond has been suggested to be an important part of the reaction coordinate. ${ }^{3,18}$ In asymmetric catalysis, such strong bonds can restrict the number of thermally accessible conformations of the base. $^{19,20}$ Conversely, the bonding geometry has to be sufficiently flexible in order to allow the Brønsted acid catalyst to reversibly bind a wide range of substrates. ${ }^{21}$ As such, the location and bonding dynamics of the proton, which report on the bond strength and geometry, affect the catalytic efficiency. ${ }^{18}$ However, most of the current understanding of such strong hydrogen bonds stems from studies of carboxylic acids. ${ }^{13,22-24}$ Hydrogen bonding involving phosphoric acid groups, as they occur in enzymatic transition ${ }^{4}$ states or in asymmetric catalysis, ${ }^{5}$ are less well studied. ${ }^{25}$

Two limiting cases are usually considered for the aggregation of a Brønsted acid with a Brønsted base: a neutral hydrogen bond with the acidic proton remaining located at the acid or a doubly ionic hydrogen bond (a strongly hydrogen-bonded ionpair), which is formed by transfer of the proton from the acid to the base. ${ }^{12,26-28}$ For example, the interaction between organophosphoric acids and imines was initially described as the coexistence of these two limiting cases. ${ }^{29}$ However, in recent years there has been evidence that proton transfer and subsequent ion-pair formation (doubly ionic hydrogen bond) dominate. $^{20,30,31}$ Yet, our previous studies have revealed an even more complex association behavior in the dipolar aprotic solvent dichloromethane: even for equimolar mixtures of phosphoric acids with imine bases, not only bimolecular aggregates (ion-pairs, Figure 1a) between the acid and the base but also multimers are formed. In the multimers a phosphoric acid donates a hydrogen bond to the deprotonated phosphoric acid anion of an ion-pair (Figure $1 \mathrm{~b}$ ). ${ }^{30,31}$ Conceptually, this multimer formation can be interpreted as an acid homodimer (Figure 1c), which are commonly observed for such mixtures, ${ }^{32-34}$ protonating the imine base. Our earlier studies

Received: May 25, 2020

Revised: July 23, 2020

Published: July 23, 2020 


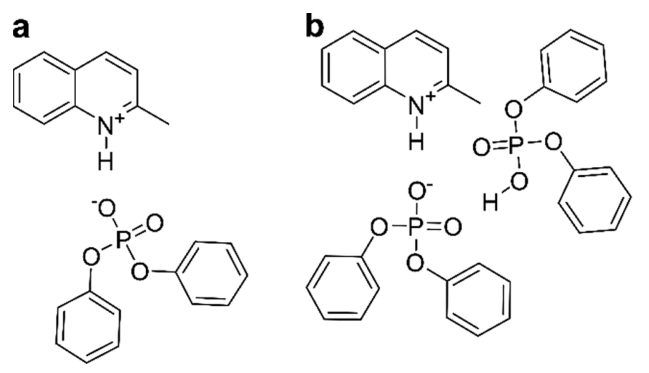

C

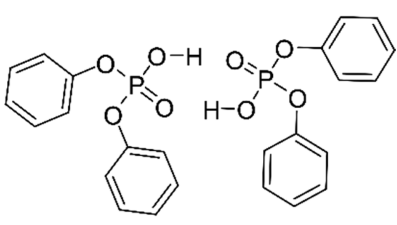

Figure 1. (a) Structure of an ion-pair consisting of one Qu and one DPP molecule. (b) For the simplest multimeric structure, i.e., a trimer, an additional DPP molecule donates a hydrogen bond to the acid anion. In panel (c) a DPP homodimer is schematically shown.

were, however, largely based on rather "slow" experimental methods (nuclear magnetic resonance (NMR) and dielectric relaxation spectroscopies (DRS)), which typically provide information on the average charge and/or proton distribution within the aggregates. As such, the different hydrogen-bonding motifs and their respective bond strengths and dynamics have remained elusive.

Linear (and nonlinear) infrared (IR) spectroscopies provide insight into the nature and strength of strong hydrogen bonds because the hydrogen-bond potential is directly reflected in the IR absorption bands. For catalytically active phosphoric acids, the vibrational frequencies have in fact been suggested to be a powerful quantity to predict catalytic activity. ${ }^{35,36}$ For strong hydrogen bonds, the anharmonic and flat potential along the proton transfer coordinate (e.g., as compared to a water-water hydrogen bond) lead, however, to rather complex infrared spectra ${ }^{13,22,26,37}$ with the absorption bands spanning more than $1000 \mathrm{~cm}^{-1}$. The width of these bands has been attributed to strong anharmonic coupling. ${ }^{16,25,38,39}$ With increasing hydrogen-bonding strength, a Fermi resonance between the proton stretching mode and the overtone of the hydrogen-bond bending vibration(s) even results in heavily mixed vibrations and an apparent splitting of the band into two main absorbances (where the absorption dip is often referred to as an Evans hole $\left.{ }^{37,39-42}\right)$. As a result of these strongly coupled vibrational modes, vibrational energy relaxation, as measured using ultrafast infrared spectroscopies, is very rapid (tens to hundreds of femtoseconds), ${ }^{13,32,39,43}$ and population of lowerfrequency modes in the course of energy relaxation results in a modulation of virtually the entire infrared spectrum. ${ }^{13}$ Yet, the energy released in the course of vibrational relaxation has been suggested to be insufficient to weaken or dissociate the aggregates. $^{13,22,44}$ Overall, the vibrational structure and dynamics of such acid-base pairs appear somewhat reminiscent of the structure and dynamics of the proton in aqueous solutions of Brønsted acids. ${ }^{45-47}$

To elucidate hydrogen bonding of catalytically relevant phosphoric acids, ${ }^{48,49}$ we study vibrational signatures of intermolecular bonding in acid-base mixtures consisting of diphenyl phosphoric acid and the base quinaldine dissolved in dichloromethane. We find the broad infrared absorption of the phosphoric acid homodimers to red-shift upon addition of the base. Density functional theory calculations suggest that the red-shift can be explained by a flatter potential along the proton transfer coordinate for hydrogen bonds in the presence of the base, as compared to the acid homodimer. To study the composition-dependent bonding dynamics, we performed IR pump-probe spectroscopy and two-dimensional IR (2D-IR) spectroscopy $^{50-54}$ experiments at different acid/base molar ratios. These experiments indicate-similar to other strong hydrogen bonds-extremely fast $(\sim 100$ fs $)$ vibrational relaxation. Yet, full equilibration of the excess energy depends on the acid-base ratio, indicative of different relaxation channels in dimers and multimers. These relaxation pathways also result in a somewhat delayed appearance of a rather uncommon off-diagonal peak in the 2D-IR spectra, most apparent for an excess of acid. We ascribe this off-diagonal signal to the population of lower energy states during vibrational relaxation, which-via strong anharmonic coupling-transiently modulate all hydrogen bonds within the molecular complexes. As such, our results highlight the collective nature of hydrogen bonding in these aggregates, which affects both hydrogen-bond strengths and energy redistribution in such acid base mixtures.

\section{MATERIALS AND METHODS}

Diphenyl phosphoric acid (diphenyl phosphate, DPP, 99\%) and quinaldine $(\mathrm{Qu}, 95 \%)$, were purchased from SigmaAldrich. Dichloromethane (DCM, 99.9\%) was purchased from Fisher Scientific. To remove residual water from DCM and $\mathrm{Qu}$, both liquids were dried over molecular sieves (4 Å). Prior to use, the liquids were filtered with a $0.2 \mathrm{~mm}$ Omnipore membrane filter (Merck) to remove solid residues from the molecular sieves. All samples were prepared by weight in volumetric glass equipment and subsequently sonicated for $\sim 5$ s. The samples were contained between two $\mathrm{CaF}_{2}$ windows $(4$ $\mathrm{mm}$ thick) separated by a $25 \mu \mathrm{m}$ PTFE spacer, except for infrared pump probe experiments, where we used $2 \mathrm{~mm}$ thick $\mathrm{CaF}_{2}$ windows separated by a $50 \mu \mathrm{m}$ PTFE spacer. For infrared pump-probe and 2D-IR spectroscopies the temperature was controlled to $22.0 \pm 0.5{ }^{\circ} \mathrm{C}$.

Fourier transform infrared spectra (FTIR) were measured using a Bruker VERTEX 70 spectrometer with $4 \mathrm{~cm}^{-1}$ resolution in transmission geometry. To obtain information on vibrational dynamics, we used broadband infrared pumpprobe experiments. In these experiments a broadband infrared pulse (centered at $\sim 2000 \mathrm{~cm}^{-1}$, full width at half-maximum (fwhm) $\sim 300 \mathrm{~cm}^{-1}$ ) excites infrared modes in the sample. The pump-induced modulation of the infrared absorption, $\Delta \alpha$, is detected with a weak, variably delayed infrared probe pulse as a function of delay time, $t$. The detected modulation of the spectra is recorded for both parallel $\left(\Delta \alpha_{\|}\right)$and perpendicular $\left(\Delta \alpha_{\perp}\right)$ polarization of the probe pulse relative to the pump pulse polarization. The isotropic (rotation-free) modulation (i.e., at the magic angle), which reflects vibrational population dynamics, is calculated as $\Delta \alpha_{\text {iso }}=\left(\Delta \alpha_{\|}+2 \Delta \alpha_{\perp}\right) / 3$. The experimental setup is described in detail elsewhere. ${ }^{55}$ Briefly, pulses centered at $800 \mathrm{~nm}(\sim 50$ fs duration, $1.5 \mathrm{~mJ}$ pulse energy, $1 \mathrm{kHz}$ repetition rate) from a Ti:sapphire regenerative amplifier (Spitfire Ace, Spectra-Physics) are used to generate 

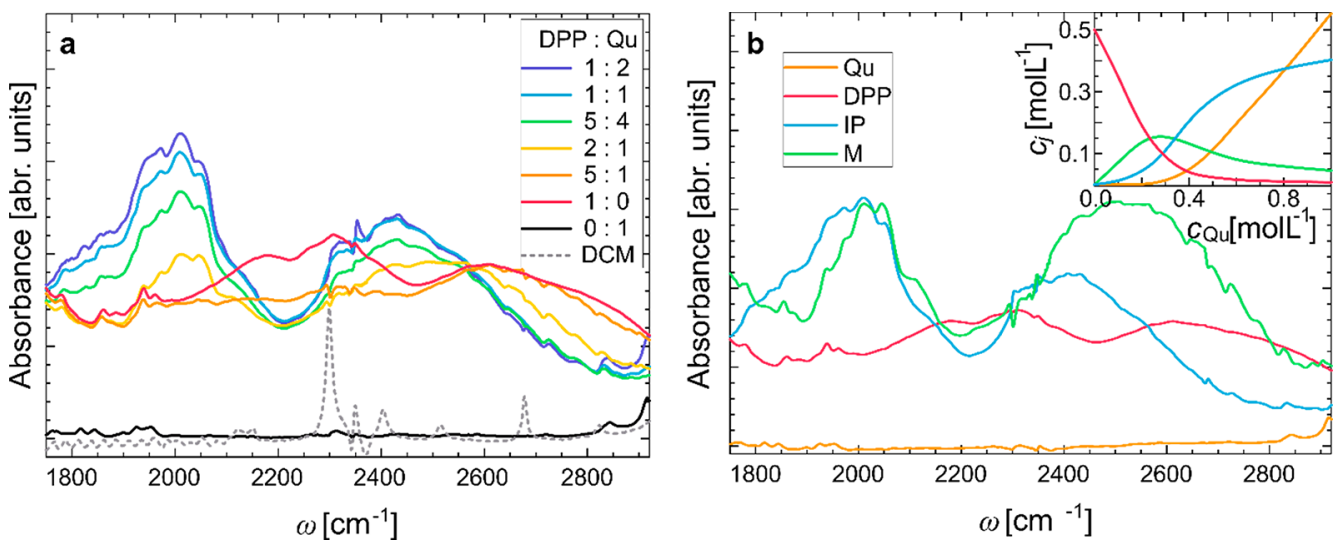

Figure 2. (a) FTIR spectra for mixtures of DPP $\left(0.5 \mathrm{~mol} \mathrm{~L}^{-1}\right)$ with varying concentrations of $\mathrm{Qu}$ in DCM. The spectrum of Qu in DCM (solid black line) shows essentially no spectral features in this frequency region. A solution of DPP in DCM (red solid line) exhibits a very broad band spanning more than $1000 \mathrm{~cm}^{-1}$ due to DPP-DPP homodimers. In mixtures of DPP and Qu two structured spectral features at $\sim 2040$ and $\sim 2500$ $\mathrm{cm}^{-1}$ are present. Contributions of the solvent (dotted gray line) have been subtracted from the mixture spectra. (b) Component spectra of $\mathrm{Qu}$, DPP homodimers (DPP), ion-pairs (IP), and multimers (M). The decomposition is based on the variation of the different species concentrations based on the equilibria reported in ref 30 (inset).

infrared pulses using an optical parametric amplifier (OPA) together with a noncollinear difference frequency generation stage (TOPAS prime \& NDFG, Light Conversion). The IR pulses $(\sim 12 \mu \mathrm{J}$ pulse energy and $\sim 100 \mathrm{fs}$ fwhm duration) are split into pump, probe, and reference beams, with the pump and the probe beams being focused into the sample. The probe beam is recollimated after the sample and, together with the reference beam, spectrally dispersed (Triax 180 spectrograph, Horiba, $100 \mathrm{l} / \mathrm{mm}$ grating) onto a $2 \times 32$ pixel mercury cadmium telluride (MCT) detector.

To resolve the spectral modulation for different pump frequencies, we perform two-dimensional infrared (2D-IR) spectroscopy experiments. ${ }^{56}$ Here the sample is excited using two temporally separated pump pulses. Variation of the coherence time $\left(t_{1}\right)$ between the two pump pulses and subsequent Fourier transformation of the transient signals at different probing frequencies provide spectral resolution along the pump axis. ${ }^{50,57-60}$ The 2D-IR experiment is based on a regenerative amplifier (Astrella, Coherent) providing $800 \mathrm{~nm}$ pulses ( 35 fs pulse duration) at a $1 \mathrm{kHz}$ repetition rate. Pulses with an energy of $2.7 \mathrm{~mJ}$ are used to pump an OPA with difference frequency generation to generate IR pulses (pulse energy $\sim 30 \mu \mathrm{J}$ ) centered at $2000 \mathrm{~cm}^{-1}$ with a $\sim 150$ fs pulse duration. The infrared pulses are guided to a commercial 2DIR spectrometer (2DQuick-IR, Phasetech Spectroscopy Inc.). Pump pulse pairs are generated using an infrared pulse-shaper, based on spectrally dispersed diffraction from a Ge-based acousto-optic modulator. ${ }^{61-63}$ The pump and the probe beams are focused and overlapped in the sample. The probe beam is then detected together with a reference beam, by dispersing both beams (using a SP2156 spectrograph, Princeton Instruments, $30 \mathrm{l} / \mathrm{mm}$ grating) onto a $128 \times 128$ pixel MCT array detector. Two-dimensional-IR spectra are recorded for different values of the waiting time.

\section{RESULTS AND DISCUSSION}

FTIR Spectra of DPP-Qu Mixtures. To study the interaction between the acid and the base at different compositions, we measure FTIR spectra of DPP- $\mathrm{Qu}$ mixtures in DCM at a constant concentration of DPP $\left(0.5 \mathrm{~mol} \mathrm{~L}^{-1}\right)$ and varying concentrations of $\mathrm{Qu}\left(0-1 \mathrm{~mol} \mathrm{~L}^{-1}\right)$. The infrared absorption spectrum of $\mathrm{Qu}$ in the $1700-2800 \mathrm{~cm}^{-1}$ spectral range (black line in Figure $2 \mathrm{a}$ ) shows no vibrational features in this spectral range. Solutions of only DPP (solid red line) exhibit a rather broad absorption spanning the full $\sim 1000 \mathrm{~cm}^{-1}$ spectral range of Figure 2a. This very broad absorption band is due to DPP homodimers, similar to what has been reported for solutions of other Brønsted acids in aprotic solvents. ${ }^{32,33}$ The remarkable width of such homodimer absorptions has already earlier been-besides different molecular conformationsrelated to strong coupling (anharmonic and excitonic) as well as Fermi resonances. ${ }^{23,25,32,64}$ Upon addition of the base $\mathrm{Qu}$ to the mixtures, we observe the emergence of two broad (more than $400 \mathrm{~cm}^{-1}$ wide), yet structured absorption features at $\sim 2040$ and $\sim 2500 \mathrm{~cm}^{-1}$ (Figure 2a, for full spectral range see Figure $\mathrm{S} 1$ in the Supporting Information). Thus, the doubly ionic $\left(\mathrm{O}^{-} \cdots \mathrm{H}-\mathrm{N}^{+}\right)$hydrogen bonds, ${ }^{28}$ which are the predominant intermolecular interaction motif for an excess of $\mathrm{Qu}$ (blue solid line in Figure 2a), give rise to similarly broad absorptions in the infrared spectra. The two spectral features are separated by a minimum in the absorbance at $\sim 2200 \mathrm{~cm}^{-1}$, which has been explained by a Fermi resonance with the hydrogen-bond bending overtone. ${ }^{16,25}$ The marked structuring of the absorbance in the presence of the base quinaldine, as opposed to the spectra in the absence of the base, shows that this Fermi resonance is most significant for ionic $\left(\mathrm{O}^{-} \cdots \mathrm{H}-\mathrm{N}^{+}\right)$ hydrogen bonds.

Close inspection of the two absorbances at $\sim 2040$ and $\sim 2500 \mathrm{~cm}^{-1}$ reveals that the amplitudes of both spectral features depend on the acid:base molar ratio. Upon increasing concentration of $\mathrm{Qu}$ the absorbance overall red-shifts (see also Figure S1, Supporting Information): While the absorbance at blue-shifted frequencies decreases (see $2810 \mathrm{~cm}^{-1}$ in Figure $\mathrm{S} 1 \mathrm{~b}$ ), the absorption at $2460 \mathrm{~cm}^{-1}$ increases by $\sim 40 \%$, and at very red-shifted frequencies by $\sim 90 \%\left(2040 \mathrm{~cm}^{-1}\right.$ in Figure $\mathrm{S} 1 \mathrm{~b})$ with increasing $\mathrm{Qu}$ concentration. These qualitative considerations suggest a redistribution of the spectral intensity between the two main absorption features at $\sim 2040$ and $\sim 2500 \mathrm{~cm}^{-1}$ depending on molar composition.

The isosbestic point at $\sim 2550 \mathrm{~cm}^{-1}$ (Figure 1a) shows that at least two molecular species give rise to observed spectra. The spectral variation at $\sim 2040$ and $\sim 2500 \mathrm{~cm}^{-1}$ for high concentrations of $\mathrm{Qu}$ suggests the presence of a third 

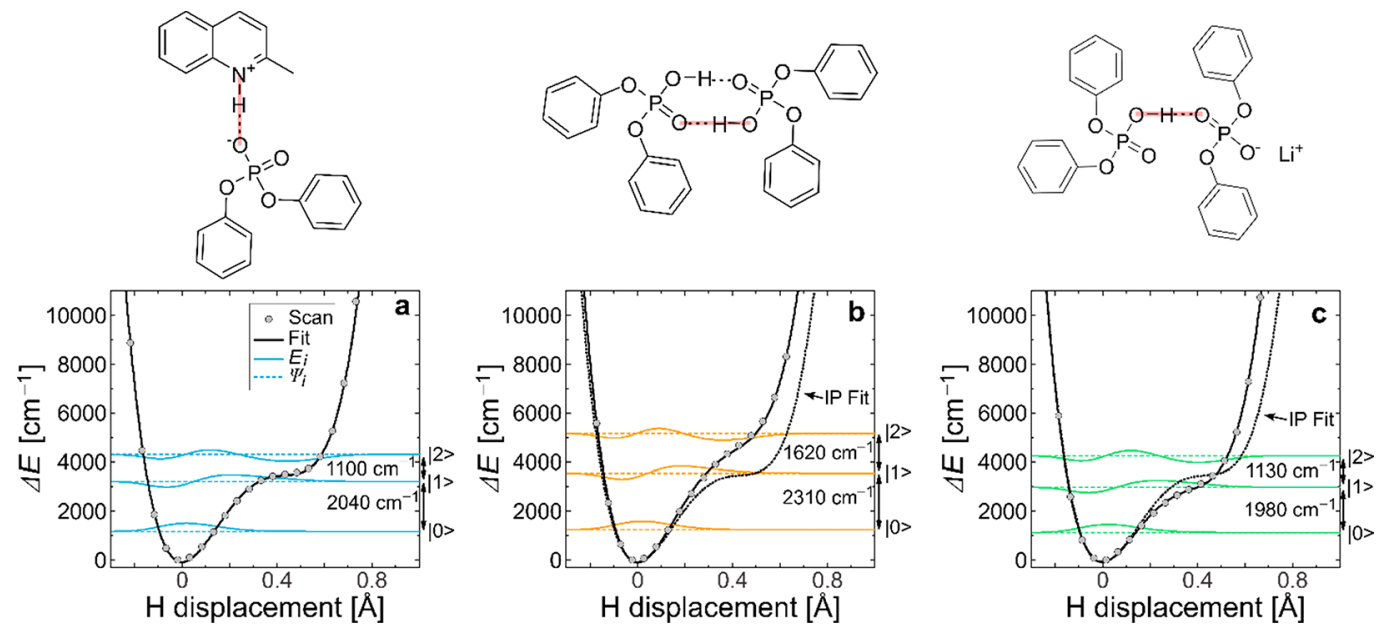

Figure 3. Potential energy profile along the proton transfer coordinate for hydrogen bonds between (a) Qu and DPP, (b) between two DPP molecules within a homodimer, and (c) between DPP and a $\mathrm{DPP}^{-}$anion with lithium as counterion. The potentials and estimated transition energies are sensitive to the $\mathrm{O}-\mathrm{O}$ or $\mathrm{O}-\mathrm{N}$ distance (molecular separation). ${ }^{16,25,65}$ Yet, calculations with fixed intermolecular distance allow for assessing the effect of variation of the hydrogen-bond acceptor. Schematic molecular structures together with the proton transfer coordinate (shaded red lines) are shown on the top of each panel. Symbols correspond to relative energies as obtained from a nonrelaxed energy scan for the hydrogen-bonding proton displaced along the $\mathrm{N}-\mathrm{O}$ (or $\mathrm{O}-\mathrm{O}$ coordinate). Black solid lines show quartic fits to the potential (see text and the Supporting Information for details). For better comparison, the potential shown in (a) is shown as dotted line in panels (b) and (c). Energy levels of the three lowest eigenstates $|0>| 1$,$\rangle , and |2\rangle$ obtained by numerically solving the one-dimensional Schrödinger equation are shown as dashed lines. The associated wave functions are depicted as solid lines and were vertically offset by the corresponding energies for clarity. Transition frequencies are highlighted with double-headed arrows.

molecular species. Previous results on the molecular complexes in solution ${ }^{30,31}$ suggested the simultaneous presence of DPP homodimers, $\mathrm{Qu}-\mathrm{DPP}$ dimers (ion-pairs with a doubly ionic $\mathrm{O}^{-} \cdots \mathrm{H}-\mathrm{N}^{+}$hydrogen bond), and (DPP) $)_{2}-\mathrm{Qu}$ multimers containing both doubly ionic $\mathrm{O}^{-} \ldots \mathrm{H}-\mathrm{N}^{+}$and singly ionic $\mathrm{P}-$ $\mathrm{O}^{-} \cdots \mathrm{H}-\mathrm{O}-\mathrm{P}$ hydrogen bonds (see Figure 1). Taking additionally the presence of free $\mathrm{Qu}$ into account, we decompose the spectra of Figure 1a into the contributions of these species. We determine the spectral contributions of $\mathrm{Qu}$, DPP homodimers, ion-pairs (IP), and multimers (M) (Figure $1)$ to the spectra in Figure 2a, using least-squares decomposition with the composition-dependent concentrations of all molecular aggregates as known covariances (see the inset of Figure $2 \mathrm{~b}$ ). The thus-extracted component spectra of $\mathrm{Qu}, \mathrm{DPP}, \mathrm{IP}$, and $\mathrm{M}$ are shown in Figure $2 \mathrm{~b}$ (for details see the Supporting Information, Figure S2). We note that using this decomposition based on molar concentrations of the species, the component spectrum for the multimers does not intersect the isosbestic point at $2550 \mathrm{~cm}^{-1}$, since $(\mathrm{DPP})_{2}-\mathrm{Qu}$ complexes contain two acidic protons as opposed to IP and DPP. The decomposition suggests that the absorbances of the ion-pairs and multimers markedly differ from the spectral contributions of the DPP homodimers. Both the ion-pairs and the multimers show a marked absorbance at $\sim 2040 \mathrm{~cm}^{-1}$, consistent with doubly ionic hydrogen bond $\mathrm{O}^{-} \cdots \mathrm{H}-\mathrm{N}^{+}$ underlying this peak. Yet, this peak is red-shifted for the ionpairs, as compared to the multimers, suggesting that association of a DPP molecule to an ion-pair to form a multimer also affects the $\mathrm{O}^{-} \cdots \mathrm{H}-\mathrm{N}^{+}$bond. The ion-pairs and the multimers show some additional absorption at $\sim 2500$ $\mathrm{cm}^{-1}$, yet for the multimers this band is more intense and broader.

Proton Transfer Potentials. The rather low (as, e.g., compared to the $\sim 3000-3500 \mathrm{~cm}^{-1}$ range for weaker hydrogen-bonded $\mathrm{O}-\mathrm{H}$ and $\mathrm{N}-\mathrm{H}$ stretching bands) resonance frequency for the proton stretching band has been explained by the proton residing in a rather shallow and anharmonic potential. ${ }^{39}$ Despite that the experimentally observed broad and structured absorbance cannot be explained by considering only a single vibrational coordinate, ${ }^{25,64,65}$ the displacement of the proton along the proton transfer coordinate has been shown to be a good estimate for the overall position of the absorbance of the hydrogen bond in the vibrational spectra. ${ }^{45-47}$ Thus, to further support the assignment to the different bonding motifs, we calculate the potential energy landscapes for the proton transfer between the $\mathrm{O}$ (or $\mathrm{N}$ ) atoms of DPP and Qu. To determine the potential of doubly ionic hydrogen bonds as in the ion-pairs and multimers $\left(\mathrm{N}-\mathrm{H}^{+} \ldots{ }^{-} \mathrm{O}\right.$, Figure $1 \mathrm{a}$ and $\left.\mathrm{b}\right)$, of neutral hydrogen bonds as in the DPP homodimers $(\mathrm{P}-\mathrm{O} \cdots \mathrm{H}-\mathrm{O}-\mathrm{P}$, Figure $1 \mathrm{c})$, and of singly ionic hydrogen bonds $\left(\mathrm{P}-\mathrm{O}^{-} \cdots \mathrm{H}-\mathrm{O}-\mathrm{P}\right.$, Figure $\left.1 \mathrm{~b}\right)$, we perform density functional theory (DFT) calculations.

The DFT calculations ${ }^{66-68}$ were performed for a $\mathrm{Qu}-\mathrm{DPP}$ pair (IP) and an electroneutral DPP-DPP pair. For the "singly ionic" $\mathrm{P}-\mathrm{O}^{-} \cdots \mathrm{H}-\mathrm{O}-\mathrm{P}$ hydrogen bonds in multimers we use an anionic $\mathrm{DPP}^{-}-\mathrm{DPP}$ pair with lithium as counterion, as a computationally feasible model system. To this end, we used the B3LYP-D3(BJ)/6-31G(d) level of theory ${ }^{69-71}$ using Grimme's D3 dispersion with Becke-Johnson damping. ${ }^{72}$ We use a polarizable continuum model for $\mathrm{DCM},{ }^{73}$ as implemented in Gaussian $16 .^{74}$ Starting from an optimized geometry (Figure S3, Supporting Information), a nonrelaxed surface scan indeed indicates a very anharmonic potential upon translating the protons along the $\mathrm{N}-\mathrm{O}$ (or $\mathrm{O}-\mathrm{O}$ ) axes (for details see Figure 3 and the Supporting Information). For the doubly ionic hydrogen bond, the global minimum of the potential corresponds to the proton located close to the nitrogen $(r(\mathrm{~N}-\mathrm{H}) \approx 1.1 \AA$ in Figure $3 \mathrm{a})$ and is separated by $\sim 3000 \mathrm{~cm}^{-1}$ from the plateau close to DPP's oxygen $(r(\mathrm{~N}-\mathrm{H})$ $\approx 1.5 \AA$ in Figure $3 \mathrm{a}$ ). The overall shape of the potential with a pronounced minimum located close to the nitrogen atom of $\mathrm{Qu}$ is similar to what has been previously reported for strong 


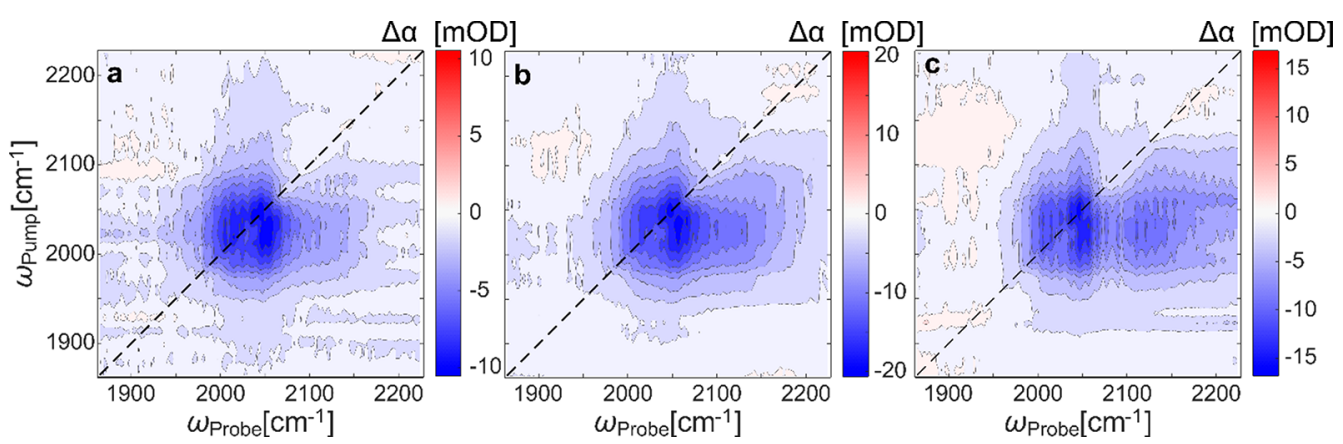

Figure 4. 2D-IR spectra at $t=50$ fs for solutions of $0.5 \mathrm{~mol} \mathrm{~L}^{-1}$ DPP and (a) 0.75 , (b) 0.5 , and (c) $0.25 \mathrm{~mol} \mathrm{~L}^{-1}$ Qu in DCM. All spectra show a bleach at $2040 \mathrm{~cm}^{-1} / 2040 \mathrm{~cm}^{-1}$, which is related to ionic hydrogen bonds. With increasing DPP concentration an off-diagonal bleaching signal at $2040 \mathrm{~cm}^{-1} / 2100 \mathrm{~cm}^{-1}$ appears.

hydrogen bonds ${ }^{12,25,26,45,53,75-77}$ and agrees well with the notion of the ionic nature of DPP-Qu dimers. ${ }^{30,31}$

To relate this potential to the vibrational transitions, we fit the potential with a quartic asymmetric double-well function as described in ref 47 (Figure 3). The corresponding onedimensional nuclear Schrödinger equation for the fitted potential was numerically solved, using the discrete variable representation method ${ }^{75}$ (see the Supporting Information for details). The thus-obtained wave functions of the three lowest lying eigenstates together with their energies are shown in Figure 3. The first vibrational excited state for the ion-pair is energetically very close to the plateau, which corresponds to the proton back transfer to the oxygen atom of DPP. As such, the proton is significantly delocalized between the acid and the base in the first vibrational excited state. The strong anharmonicity of the potential also results in a very large difference in transition energies between the lowest lying states: $|0>-| 1>\sim 2040 \mathrm{~cm}^{-1}$ vs $|1>-| 2>\sim 1100 \mathrm{~cm}^{-1}$, with the fundamental transition in qualitative agreement with the experimentally observed bands for an excess of $\mathrm{Qu}$ in Figure $2 a$.

For the electroneutral hydrogen bond in DPP homodimers (Figure $3 b$ ), we find the proton transfer potential to be steeper, resulting in the vibrational transitions to be located at $\sim 2310$ $\mathrm{cm}^{-1}(|0>-| 1>)$ and at $\sim 1620 \mathrm{~cm}^{-1}(|1>-| 2>)$. We note that for the slightly nonlinear $\mathrm{O}-\mathrm{H}-\mathrm{O}$ bond of the DPP homodimers, the results are sensitive to the exact choice of the coordinate: translating the proton along the $\mathrm{O}-\mathrm{H}$ axis results in an increase of the transition frequencies by up to 160 $\mathrm{cm}^{-1}$ (see Figure S4, Supporting Information). The potential of a singly ionic hydrogen bond, which we model using a $\mathrm{Li}^{+}$ $\mathrm{DPP}^{-}$DPP complex (Figure 3c), is comparable to the potential of the doubly ionic hydrogen bond and only differs for large proton displacements. Thus, the calculated transition frequencies are similar to those of the doubly ionic hydrogen bond and markedly lower than those of the electroneutral hydrogen bonds in DPP homodimers.

Overall, the thus-calculated transition energies are in broad agreement with the experimentally observed overall red-shift of the absorbance upon the addition of $\mathrm{Qu}$ (Figure 2a) and the spectral decomposition in Figure $2 \mathrm{~b}$. In the absence of $\mathrm{Qu}$, the DPP homodimers give rise to spectral contributions at higher frequencies (see Figure 2b), in line with the transition energy of the DPP-DPP homodimers (Figure 3b). For an excess of $\mathrm{Qu}$, ion-pairs dominate, for which the proton transfer is suggested to give rise to spectral contributions as low as 2000 $\mathrm{cm}^{-1}$ (Figure 3a). Also the formation of singly ionic hydrogen bonds contributes to the gradual shift of the spectrum as shown in Figure 2a.

Composition-Dependent Vibrational Couplings. We use 2D-IR spectroscopy to elucidate the effect of these different hydrogen-bonding motifs on the vibrational dynamics of solutions of $\mathrm{Qu}$ and DPP. In 2D-IR spectroscopy the modulation of the infrared absorbance of the sample is probed (probe frequency, $\omega_{\text {Probe }}$ ) as a function of (excitation) pump frequency, $\omega_{\text {Pump }}$. Vibrational dynamics are detected by varying the delay time, $t$, between the pump and the probe pulses. Commonly, for each vibrational mode a pair of peaks is observed in the 2D-IR spectra: a negative peak on the diagonal $\left(\omega_{\text {Pump }}=\omega_{\text {Probe }}\right)$ and a positive peak at red-shifted probe frequencies $\left(\omega_{\text {Pump }}>\omega_{\text {Probe }}\right)$. The former results from ground state depletion and stimulated emission, while the positive peak is due to excited state absorption of the anharmonic vibrational mode. Vibrational coupling (and/or chemical exchange/energy transfer) between distinct vibrational modes typically gives rise to two instantaneous (or delayed) offdiagonal peaks. The position of such off-diagonal peaks is symmetric with respect to the diagonal. ${ }^{50,51,54,57,78}$

In Figure 4 we show the absorptive 2D-IR spectra for mixtures of DPP and $\mathrm{Qu}$ with varying molar ratios at $t=50 \mathrm{fs}$ (see Figure 4a). For all spectra we observe a bleaching signal on the diagonal, centered at $2040 \mathrm{~cm}^{-1}$. Even at this early delay time ( $50 \mathrm{fs}$ ) the signal is already fairly round, and we find no significant elongation along the diagonal. This round shape indicates that no inhomogeneous broadening is detectable within our time resolution ( $\sim 150 \mathrm{fs})$. Thus, either the peak at $2040 \mathrm{~cm}^{-1}$ is homogeneously broadened or-if also inhomogeneously broadened-spectral diffusion is very fast. Spectral diffusion due to breaking and reformation of these strong hydrogen bonds is unlikely to occur on such a fast time scale, given the size of the hydrogen-bonded molecules. Yet, coupling to or mixing with lower frequency modes, like, e.g., hydrogen-bond bending vibrations ${ }^{16}$ or librations of the solvent, ${ }^{79}$ can explain the decay of frequency-frequency correlation on a subpicosecond time scale. Despite the round shape of the on-diagonal peak at $2040 \mathrm{~cm}^{-1}$, the overall transient signal is somewhat structured, with two minima on the diagonal at $\sim 2010$ and $\sim 2040 \mathrm{~cm}^{-1}$ (see the diagonal cut in Figure S5, Supporting Information). As this structure is also present in the FTIR spectra (Figure 2), the clear structuring in the nonlinear infrared spectra may point toward vibrational progression with a $\left(\sim 30 \mathrm{~cm}^{-1}\right)$ lower frequency vibration, similar to what has been observed for acid homodimers. ${ }^{80}$ Remarkably, at all time delays we do not detect an excited state 

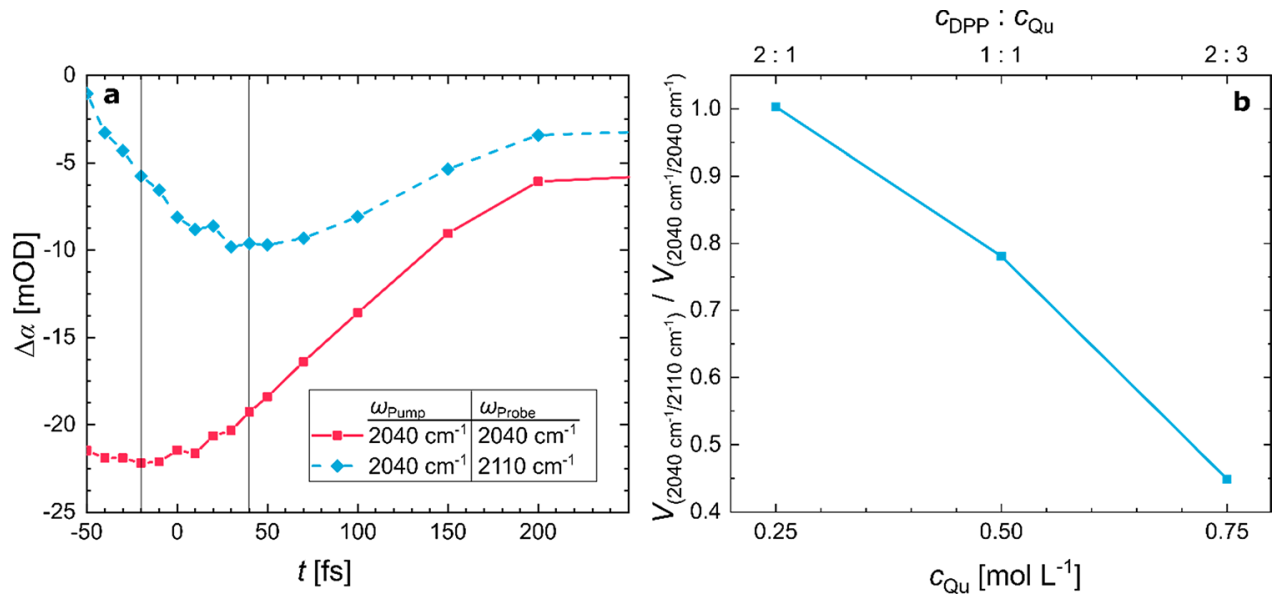

Figure 5. (a) Time traces of selected 2D-IR peaks $\left(2040 \mathrm{~cm}^{-1} / 2040 \mathrm{~cm}^{-1}\right)$ and $\left(2040 \mathrm{~cm}^{-1} / 2110 \mathrm{~cm}^{-1}\right)$ for an equimolar mixture of DPP and Qu in DCM. A 60 fs delayed appearance of the maximum bleach for the $2040 \mathrm{~cm}^{-1} / 2110 \mathrm{~cm}^{-1}$ trace, relative to the $2040 \mathrm{~cm}^{-1} / 2040 \mathrm{~cm}^{-1}$ trace, can be observed. The two vertical solid gray lines mark the maximum bleaching of both signals. (b) Ratio of the volume integrals of the off-diagonal peak to the on-diagonal peak vs concentration of $\mathrm{Qu}$ at $t=50 \mathrm{fs}$. The integrals taken at pump frequencies from 1980 to $2083 \mathrm{~cm}{ }^{-1}$ and probe frequencies $1980-2083 \mathrm{~cm}^{-1}$ or $2083-2230 \mathrm{~cm}^{-1}$ evidence the increase of the $2040 \mathrm{~cm}^{-1} / 2100 \mathrm{~cm}^{-1}$ peak with increasing DPP to Qu ratio. Lines connecting the symbols are to a guide the eye.

absorption adjacent to the bleaching signal at red-shifted probe frequencies. The absence of such detectable positive signals is consistent with our DFT calculations, which predict the excited state absorptions at $\sim 1000 \mathrm{~cm}^{-1}$ (Figure 3), well outside our detection range. Additionally, rapid relaxation of the first vibrational excited state, which will be discussed below, can result in a significant attenuation of the excited state absorption.

In contrast to the absence of a detectable off-diagonal peak due to the excited state absorption, we find at all molar ratios the predominant bleaching signal to be elongated toward higher probe frequencies: depending on molar composition a more or less intense blue-shifted bleaching signal at $\omega_{\text {Pump }} /$ $\omega_{\text {Probe }}=2040 \mathrm{~cm}^{-1} / 2100 \mathrm{~cm}^{-1}$ adjacent to the transient signal at the diagonal is present. The fact that we do not detect corresponding spectral intensity on the diagonal $\left(2100 \mathrm{~cm}^{-1} /\right.$ $2100 \mathrm{~cm}^{-1}$ ) suggests that the transition dipole moment of the modes giving rise to the signal at $\omega_{\text {Probe }}=2100 \mathrm{~cm}^{-1}$ is too low to be directly excited in the 2D-IR experiment. Also the absence of a corresponding transient signal above the diagonal (symmetric with respect to the diagonal at $2100 \mathrm{~cm}^{-1} / 2040$ $\mathrm{cm}^{-1}$ ) renders coupling, chemical exchange, or resonant energy transfer as the origin of this feature unlikely as these mechanisms would lead to a second peak above the diagonal. Thus, the signal must have different origins. A possible explanation for the appearance of such transient bleaching signals only below the diagonal is transient modulation of vibrational modes in the course of vibrational relaxation: vibrational relaxation of initially excited modes (transiently) populates lower-frequency modes. If some of these lower frequency modes are strongly coupled-similar to other strong hydrogen bonds ${ }^{13,22,23,81}$ - to the modes that give rise to the weak, yet detectable, absorbance in the FTIR spectra at $\sim 2100$ $\mathrm{cm}^{-1}$ (Figure 2a), an off-diagonal peak appears.

Since this scenario results in off-diagonal contributions to the spectra only after vibrational relaxation, one may expect a delayed appearance of the transient signal below the diagonal. Despite our pulse duration being $\sim 150$ fs and the contribution of different "artifacts" 50 at such early time delays, the time traces in Figure 5a may indeed indicate that the signal below the diagonal appears delayed relative to the signal on the diagonal. The observed $60 \mathrm{fs}$ delay is in fact similar to the vibrational relaxation time of the on-diagonal peak (see discussion below). As such, also the temporal evolution of the signals supports the above-described scenario: After vibrational relaxation on a $\sim 100$ fs time scale, a manifold of lower frequency vibrational states is transiently populated, which modulates the spectral signatures ${ }^{23,32,82}$ of both the initially excited modes underlying the peak at $2040 \mathrm{~cm}^{-1}$ and the modes giving rise to the absorbance at $\sim 2100 \mathrm{~cm}^{-1}$ (Figure 2 ). It is important to note that in the studied concentration range, where molecular aggregates between the base and acid molecule(s) are separated from each other by solvent molecules, the vibrational energy is initially (within $\sim 100 \mathrm{fs}$ ) distributed over lower energy modes of molecules in spatial proximity. Conversely, relaxation and dissipation of the energy to low-frequency modes of more distant molecules typically occur on much longer time scales. ${ }^{55,83,84}$ As such, the delayed, though very fast, appearance of the bleaching signal at $\omega_{\text {Probe }} \sim$ $2100 \mathrm{~cm}^{-1}$ suggests that after excitation of the vibrations at $2040 \mathrm{~cm}^{-1}$ the transiently populated lower frequency modes are localized within the same molecular aggregate.

The decomposed FTIR spectra, which indicate a shoulder at $2100 \mathrm{~cm}^{-1}$ for the multimers (see Figure $2 \mathrm{~b}$ ), suggest the multimers being related to the off-diagonal peak in the 2D-IR spectra. In line with this notion, the negative signal at 2040 $\mathrm{cm}^{-1} / 2100 \mathrm{~cm}^{-1}$ in the 2D-IR spectra in Figure 4 is weak for an excess of base, where multimers are the minor species, and becomes more pronounced for an excess of acid, at which more multimers are formed. To quantify the intensity of this off-diagonal, we determine the volume integrals of both signals at $\left(\sim 2040 \mathrm{~cm}^{-1} / \sim 2100 \mathrm{~cm}^{-1}\right)$ and $\left(\sim 2040 \mathrm{~cm}^{-1} / \sim 2040\right.$ $\mathrm{cm}^{-1}$ ). The ratios of the integrated intensities (over $\omega_{\text {Pump }}=$ $1980-2083 \mathrm{~cm}^{-1}$ and $\omega_{\text {Probe }}=1980-2083$ or $2083-2230$ $\mathrm{cm}^{-1}$ ) in Figure $5 \mathrm{~b}$ show a marked correlation with the concentration of base, indicating that the spectral signature below the diagonal correlates with the excess of acid. We note that for an excess of acid also DPP homodimers are present (Figure 2a), yet for solutions of only DPP, we find no detectable signals (see Figure S6, Supporting Information). 

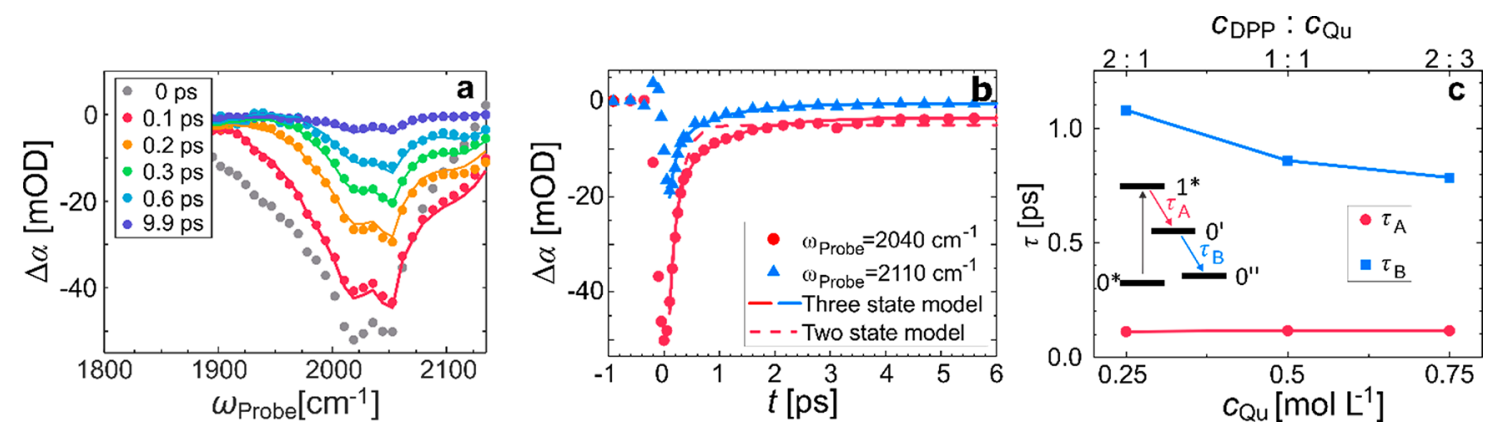

Figure 6. (a) Transient infrared spectra of an equimolar mixture of $\mathrm{Qu}$ and DPP showing a structured peak at $\sim 2040 \mathrm{~cm}^{-1}$ and a shoulder at $\sim 2100 \mathrm{~cm}^{-1}$, which appears $\sim 100 \mathrm{fs}$ delayed. (b) Delay traces at selected probing frequencies for an equimolar mixture of DPP and Qu in DCM. Symbols in panels (a) and (b) show experimental data, and solid lines show the fits with the three-state kinetic model (see also the Supporting Information). The dashed red line in panel (b) shows the fit with a two-state model at $\omega_{\text {Probe }}=2040 \mathrm{~cm}^{-1}$. (c) Relaxation times as a function of concentration of $\mathrm{Qu}$, as extracted from fitting the kinetic model (see the inset of panel c) to the transient data.

This renders the signal below the diagonal in Figure 4 to be due to acid homodimers unlikely. Conversely, the singly ionic $\mathrm{P}-\mathrm{O}^{-} \ldots \mathrm{H}-\mathrm{O}-\mathrm{P}$ hydrogen bonds in the multimers are in spatial proximity to the doubly ionic $\mathrm{O}^{-} \cdots \mathrm{H}-\mathrm{N}^{+}$hydrogen bonds (see also Figure 1), and our DFT calculations indicate that their transition frequencies are rather similar (Figure 3a and 3c). Hence, the appearance of the signal at $\sim 2040 \mathrm{~cm}^{-1} /$ $\sim 2100 \mathrm{~cm}^{-1}$ can be explained by excitation and relaxation of the $\mathrm{O}^{-} \cdots \mathrm{H}-\mathrm{N}^{+}$hydrogen bond at $\sim 2040 \mathrm{~cm}^{-1}$ after which the dissipated excess energy gives rise to a transient modulation of the $\mathrm{P}-\mathrm{O}^{-} \cdots \mathrm{H}-\mathrm{O}-\mathrm{P}$ hydrogen bonds (and the $\mathrm{O}^{-} \cdots \mathrm{H}-\mathrm{N}^{+}$ hydrogen bonds) within multimers. As such, our results provide evidence for the frequently inferred strong coupling $^{23,25,32,64}$ for acid-base hydrogen bonds, also modulating hydrogen bonds in the direct proximity; i.e., the hydrogen bonds are highly collective.

Effect of Composition on Vibrational Relaxation Pathways. To explore the consequences of these composition-dependent couplings on vibrational energy relaxation pathways, we investigate the vibrational dynamics of $\mathrm{Qu}-\mathrm{DPP}$ mixtures in more detail. As the 2D-IR spectra exhibit very little dependence on the pump frequency, we explore the vibrational dynamics based on broadband IR pump-probe experiments at $1880-2135 \mathrm{~cm}^{-1}$ (for experiments centered at $\sim 2500 \mathrm{~cm}^{-1}$ see Figures S7 and S8, Supporting Information). In an IR pump-probe experiment the transient signals are recorded as a function of delay time; in contrast to a 2D-IR experiment, the sample's response is integrated over all pump frequencies. The thus-obtained transient spectra at early time delays (Figure 6a) resemble the spectral signatures (dominant bleach at $\sim 2040$ $\mathrm{cm}^{-1}$ and a shoulder above $\sim 2100 \mathrm{~cm}^{-1}$ ) as seen in the $2 \mathrm{D}$-IR experiments. Similar to the $2 \mathrm{D}$-IR experiments, also in the pump-probe experiments the maximum bleaching signal shows a double-peak structure, and the maximum bleaching signal at $\sim 2100 \mathrm{~cm}^{-1}$ is somewhat delayed (Figure $6 \mathrm{a}$ ).

As can be seen from the delay traces in Figure $6 \mathrm{~b}$ and Figure S9, the transient signals rapidly decay with increasing time delay, indicative of fast vibrational energy relaxation. At $t>5$ ps the signals plateau, and a persistent, weak modulation arises from a heated ground state, for which the spectral modulation results from a slight local rise in temperature. To quantitatively extract the vibrational relaxation dynamics, we fit a kinetic relaxation model (see the Supporting Information for more details) to the transient signals (Figure 6c): ${ }^{85}$ In this model, infrared excitation populates a vibrationally excited state, $1 *$. Upon relaxation with a relaxation time $\tau_{\mathrm{A}}$ an intermediate state, $0^{\prime}$, is transiently populated, which further relaxes with a relaxation time $\tau_{\mathrm{B}}$ to a heated ground state, $0^{\prime \prime}$. We note that in this model, the states $0^{\prime}$ and $0^{\prime \prime}$ subsume the manifold of states over which the vibrational energy is distributed. For instance, the thermal state 0 " contains all states that are populated upon increase of the temperature after reaching a local thermal equilibrium. We assume the same population dynamics at all probe frequencies, with different transient spectra for the three states (i.e., different spectral contributions of the three states, see Figure S10). Similar to previous reports on the relaxation of strongly hydrogen-bonded complexes, ${ }^{13,51}$ this three-state model can describe the experimental data with the least number of adjustable parameters: As can be seen from the solid lines in Figure 6a and b, the model excellently describes the experimental data for the DPP-Qu mixtures at $t>0.1 \mathrm{ps}$, while a two-state model cannot fully capture the dynamics at $0.1 \leq t / \mathrm{ps}<5$ (see the red dashed line in Figure $6 \mathrm{~b}$ ). From this model, we find the initial relaxation time $\tau_{\mathrm{A}}$ to be very fast, $\sim 100$ fs (Figure 6c). Such short relaxation time is common to strongly hydrogen-bonded complexes ${ }^{13,51}$ and is again indicative of strong (anharmonic) coupling to lower-frequency modes, as the coupling provides efficient transfer paths for the excess vibrational energy. Yet, we find that the acid-base composition hardly affects these dynamics (Figure 6c).

In contrast to the composition-independent values of $\tau_{\mathrm{A}}$, we find that $\tau_{\mathrm{B}}$-the relaxation from the intermediate state $0^{\prime}-$ depends on the molar ratio of acid and base (Figure $6 \mathrm{c}$ ). With increasing concentration of $\mathrm{Qu}, \tau_{\mathrm{B}}$ becomes shorter $\left(\tau_{\mathrm{B}} \approx 0.8\right.$ ps at $\left.c_{\mathrm{Qu}}=0.75 \mathrm{~mol} \mathrm{~L}^{-1}\right)$, whereas the relaxation is slower for an excess of the acid $\left(\tau_{\mathrm{B}} \approx 1.1 \mathrm{ps}\right.$ at $\left.c_{\mathrm{Qu}}=0.25 \mathrm{~mol} \mathrm{~L}^{-1}\right)$. This variation of the relaxation rate suggests that the formation of multimers affects vibrational energy relaxation pathways: With increasing acid concentration the vibrational energy is contained within the excited molecular complex for a longer time. This is again in broad concordance with vibrational coupling within multimers being markedly different than in dimers as concluded from the 2D-IR experiments.

\section{CONCLUDING REMARKS}

We report on the composition-dependent vibrational structure and dynamics of hydrogen-bonded complexes consisting of the base quinaldine and diphenyl phosphoric acid. At all compositions we find broad absorption features in the infrared absorption spectra spanning more than $1000 \mathrm{~cm}^{-1}$ - common to strongly hydrogen-bonded complexes. Upon addition of quinaldine to a solution of diphenyl phosphoric acid, the 
infrared absorption gradually red-shifts to lower frequencies, and for an excess of base the observed absorbance peaks at $\sim 2040 \mathrm{~cm}^{-1}$. Spectral decomposition and DFT calculations show that the formation of doubly ionic hydrogen bonds between DPP and $\mathrm{Qu}$ and singly ionic hydrogen bond between $\mathrm{DPP}$ and $\mathrm{DPP}^{-}$give rise to this red-shift. The associated proton potentials of these bonds are very anharmonic: within the doubly ionic hydrogen bonds the proton is localized near the base in its vibrational ground state but becomes more delocalized in the first excited state. Thus, the width of the absorption spectrum can be attributed to anharmonic coupling of the proton vibrations to other modes together with thermally induced fluctuations.

Ultrafast infrared experiments reveal very fast vibrational relaxation on a $\sim 100 \mathrm{fs}$ time scale after excitation at $\sim 2000$ $\mathrm{cm}^{-1}$. This fast vibrational relaxation goes along with (transient) population of lower-frequency vibrational modes, which give rise to longer-lived $(0.8-1.1 \mathrm{ps})$ spectral modulations. A delayed off-diagonal peak in the 2D-IR spectra, which is most pronounced for an excess of acid, suggests that some of these lower-frequency vibrational modes are strongly coupled to both the ${ }^{+} \mathrm{N}-\mathrm{H} \cdots{ }^{-} \mathrm{O}-\mathrm{P}$ hydrogen bond and the $\mathrm{P}-\mathrm{O}^{-} \ldots \mathrm{H}-\mathrm{O}-\mathrm{P}$ hydrogen bond in multimers. The composition-dependent coupling also affects energy relaxation pathways, and our results suggest that the vibrational energy remains more localized within the multimers over longer times, as compared to the hydrogen-bonded dimers/ion-pairs, as evidenced by the longer equilibration time $\tau_{\mathrm{B}}$ for an excess of DPP.

As such, our results indicate that the acid:base ratio affects the vibrational structure and dynamics. Even for equimolar mixtures, where one could intuitively assume solely 1:1 aggregation, formation of trimers and/or larger multimers results in additional vibrational couplings and dynamics that differ from those of isolated acid-base dimers. More generally, our results indicate a composition dependence of the very fast proton dynamics in strong, doubly ionic hydrogen-bonded aggregates of DPP and quinaldine, which are relevant to Brønsted acid organocatalysis. Fast vibrational relaxation and strong coupling of the studied vibrational modes show that the doubly ionic hydrogen bond can efficiently dissipate excess energy from $\mathrm{Qu}$ into lower-frequency modes. Coordination of an additional DPP molecule to form a trimer alters hydrogenbond strengths, energy transfer paths, and transfer rates. These altered properties of the acidic site may be also relevant to substrate activation and transport of energy upon chemical conversion for catalyses involving two acidic groups, where the combination of two acidic moieties can enhance catalytic efficiency. ${ }^{86}$ Overall, our findings highlight a high degree of collectivity of the hydrogen bonds in such multimeric aggregates.

\section{ASSOCIATED CONTENT}

\section{SI Supporting Information}

The Supporting Information is available free of charge at https://pubs.acs.org/doi/10.1021/acs.jpcb.0c04714.

Full spectral range infrared absorption spectra and details of the component decomposition; details of the DFT calculations and calculations of the vibrational transitions; additional pump-probe experiments together with details of the kinetic modeling of the infrared pump-probe data (PDF)

\section{AUTHOR INFORMATION}

\section{Corresponding Author}

Johannes Hunger - Max-Planck-Institute for Polymer Research, 55128 Mainz, Germany; 이이.org/0000-0002-4419-

5220; Email: hunger@mpip-mainz.mpg.de

\section{Authors}

Christian Malm - Max-Planck-Institute for Polymer Research, 55128 Mainz, Germany; 이이.org/0000-0002-6005-0611

Leon A. Prädel - Max-Planck-Institute for Polymer Research, 55128 Mainz, Germany

Bogdan A. Marekha - Max-Planck-Institute for Polymer Research, 55128 Mainz, Germany; 이이.org/0000-00033142-8029

Maksim Grechko - Max-Planck-Institute for Polymer Research, 55128 Mainz, Germany; 이이. orcid.org/0000-0002-7717$387 \mathrm{X}$

Complete contact information is available at:

https://pubs.acs.org/10.1021/acs.jpcb.0c04714

\section{Notes}

The authors declare no competing financial interest.

\section{ACKNOWLEDGMENTS}

We thank Mischa Bonn, Ellen H. G. Backus, Heejae Kim, and Christoph Bernhard for fruitful discussion. B.A.M. gratefully acknowledges financial support from the Alexander von Humboldt foundation. This project has received funding from the European Research Council (ERC) under the European Union's Horizon 2020 research and innovation program (grant agreement No. 714691).

\section{REFERENCES}

(1) Gilli, P.; Pretto, L.; Bertolasi, V.; Gilli, G. Predicting HydrogenBond Strengths from Acid-Base Molecular Properties. The pKa Slide Rule: Toward the Solution of a Long-Lasting Problem. Acc. Chem. Res. 2009, 42, 33-44.

(2) Petersen, P. B.; Roberts, S. T.; Ramasesha, K.; Nocera, D. G.; Tokmakoff, A. Ultrafast N-H Vibrational Dynamics of Cyclic Doubly Hydrogen-Bonded Homo- and Heterodimers. J. Phys. Chem. B 2008, 112, 13167-13171.

(3) Carra, C.; Iordanova, N.; Hammes-Schiffer, S. Proton-Coupled Electron Transfer in a Model for Tyrosine Oxidation in Photosystem II. J. Am. Chem. Soc. 2003, 125, 10429-10436.

(4) Perrin, C. L.; Nielson, J. B. Strong" Hydrogen Bonds in Chemistry and Biology. Annu. Rev. Phys. Chem. 1997, 48, 511-544.

(5) Parmar, D.; Sugiono, E.; Raja, S.; Rueping, M. Complete Field Guide to Asymmetric BINOL-Phosphate Derived Brønsted Acid and Metal Catalysis: History and Classification by Mode of Activation; Brønsted Acidity, Hydrogen Bonding, Ion Pairing, and Metal Phosphates. Chem. Rev. 2014, 114, 9047-9153.

(6) Skubi, K. L.; Blum, T. R.; Yoon, T. P. Dual Catalysis Strategies in Photochemical Synthesis. Chem. Rev. 2016, 116, 10035-10074.

(7) Akiyama, T. Stronger Brønsted Acids. Chem. Rev. 2007, 107, 5744-5758.

(8) Hadži, D. Infrared Spectra of Strongly Hydrogen-Bonded Systems. Pure Appl. Chem. 1965, 11, 435-453.

(9) Zundel, G. Hydrogen Bonds with Large Proton Polarizability and Proton Transfer Processes in Electrochemistry and Biology. In Advances in Chemical Physics; Prigogine, I., Rice, S. A., Eds.; John Wiley \& Sons: New York, 2007; Vol. 111, pp 1-217.

(10) Eigen, M. Proton Transfer, Acid-Base Catalysis, and Enzymatic Hydrolysis. Part I: Elementray Processes. Angew. Chem., Int. Ed. 1964, $3,1-19$. 
(11) Szafran, M. Recent Aspects of the Proton Transfer Reaction in H-Bonded Complexes. J. Mol. Struct. 1996, 381, 39-64.

(12) Koeppe, B.; Pylaeva, S. A.; Allolio, C.; Sebastiani, D.; Nibbering, E. T. J.; Denisov, G. S.; Limbach, H.-H.; Tolstoy, P. M. Polar Solvent Fluctuations Drive Proton Transfer in Hydrogen Bonded Complexes of Carboxylic Acid with Pyridines: NMR, IR and Ab Initio MD Study. Phys. Chem. Chem. Phys. 2017, 19, 1010-1028.

(13) Stingel, A. M.; Petersen, P. B. Couplings Across the Vibrational Spectrum Caused by Strong Hydrogen Bonds: A Continuum 2D IR Study of the 7-Azaindole-Acetic Acid Heterodimer. J. Phys. Chem. B 2016, 120, 10768-10779.

(14) Saunders, L. K.; Nowell, H.; Hatcher, L. E.; Shepherd, H. J.; Teat, S. J.; Allan, D. R.; Raithby, P. R.; Wilson, C. C. Exploring Short Strong Hydrogen Bonds Engineered in Organic Acid Molecular Crystals for Temperature Dependent Proton Migration Behaviour Using Single Crystal Synchrotron X-Ray Diffraction (SCSXRD). CrystEngComm 2019, 21, 5249-5260.

(15) Asbury, J. B.; Steinel, T.; Stromberg, C.; Gaffney, K. J.; Piletic, I. R.; Fayer, M. D. Hydrogen Bond Breaking Probed with Multidimensional Stimulated Vibrational Echo Correlation Spectroscopy. J. Chem. Phys. 2003, 119, 12981-12997.

(16) Van Hoozen, B. L.; Petersen, P. B. A Combined Electronic Structure and Molecular Dynamics Approach to Computing the $\mathrm{OH}$ Vibrational Feature of Strongly Hydrogen-Bonded Carboxylic Acids. J. Chem. Phys. 2017, 147, 224304.

(17) van den Broek, M. A. F. H.; Kropman, M. F.; Bakker, H. J. Ultrafast Pump-Probe Spectroscopy of Strongly Hydrogen-Bonded Hydrogen Fluoride-Pyridine Complexes. Chem. Phys. Lett. 2002, 357, $8-14$.

(18) Maji, R.; Mallojjala, S. C.; Wheeler, S. E. Chiral Phosphoric Acid Catalysis: From Numbers to Insights. Chem. Soc. Rev. 2018, 47, $1142-1158$.

(19) Overvoorde, L. M.; Grayson, M. N.; Luo, Y.; Goodman, J. M. Mechanistic Insights into a BINOL-Derived Phosphoric AcidCatalyzed Asymmetric Pictet-Spengler Reaction. J. Org. Chem. 2015, 80, 2634-2640.

(20) Sorgenfrei, N.; Hioe, J.; Greindl, J.; Rothermel, K.; Morana, F.; Lokesh, N.; Gschwind, R. M. NMR Spectroscopic Characterization of Charge Assisted Strong Hydrogen Bonds in Brønsted Acid Catalysis. J. Am. Chem. Soc. 2016, 138, 16345-16354.

(21) Greindl, J.; Hioe, J.; Sorgenfrei, N.; Morana, F.; Gschwind, R. M. Brønsted Acid Catalysis-Structural Preferences and Mobility in Imine/Phosphoric Acid Complexes. J. Am. Chem. Soc. 2016, 138, 15965-15971.

(22) Gündoğdu, K.; Bandaria, J.; Nydegger, M.; Rock, W.; Cheatum, C. M. Relaxation and Anharmonic Couplings of the O-H Stretching Vibration of Asymmetric Strongly Hydrogen-Bonded Complexes. J. Chem. Phys. 2007, 127, 044501.

(23) Stingel, A. M.; Calabrese, C.; Petersen, P. B. Strong Intermolecular Vibrational Coupling through Cyclic HydrogenBonded Structures Revealed by Ultrafast Continuum Mid-IR Spectroscopy. J. Phys. Chem. B 2013, 117, 15714-15719.

(24) Smirnov, S. N.; Golubev, N. S.; Denisov, G. S.; Benedict, H.; SchahMohammedi, P.; Limbach, H.-H. H. Hydrogen Deuterium Isotope Effects on the NMR Chemical Shifts and Geometries of Intermolecular Low-Barrier Hydrogen-Bonded Complexes. J. Am. Chem. Soc. 1996, 118, 4094-4101.

(25) Van Hoozen, B. L.; Petersen, P. B. Vibrational Tug-of-War: The pKa Dependence of the Broad Vibrational Features of Strongly Hydrogen-Bonded Carboxylic Acids. J. Chem. Phys. 2018, 148, 134309.

(26) Intermolecular Forces; Huyskens, P. L., Luck, W. A. P., ZeegersHuyskens, T., Eds.; Springer: Berlin, Heidelberg, 1991.

(27) Ashworth, C. R.; Matthews, R. P.; Welton, T.; Hunt, P. A. Doubly Ionic Hydrogen Bond Interactions within the Choline Chloride-Urea Deep Eutectic Solvent. Phys. Chem. Chem. Phys. 2016, 18, 18145-18160.

(28) Hunt, P. A.; Ashworth, C. R.; Matthews, R. P. Hydrogen Bonding in Ionic Liquids. Chem. Soc. Rev. 2015, 44, 1257-1288.
(29) Fleischmann, M.; Drettwan, D.; Sugiono, E.; Rueping, M.; Gschwind, R. M. Brønsted Acid Catalysis: Hydrogen Bonding versus Ion Pairing in Imine Activation. Angew. Chem., Int. Ed. 2011, 50, 6364-6369.

(30) Malm, C.; Kim, H.; Wagner, M.; Hunger, J. Complexity in Acid-Base Titrations: Multimer Formation Between Phosphoric Acids and Imines. Chem. - Eur. J. 2017, 23, 10853-10860.

(31) Kim, H.; Sugiono, E.; Nagata, Y.; Wagner, M.; Bonn, M.; Rueping, M.; Hunger, J. Role of Ion-Pairs in Brønsted Acid Catalysis. ACS Catal. 2015, 5, 6630-6633.

(32) Heyne, K.; Huse, N.; Dreyer, J.; Nibbering, E. T. J.; Elsaesser, T.; Mukamel, S. Coherent Low-Frequency Motions of Hydrogen Bonded Acetic Acid Dimers in the Liquid Phase. J. Chem. Phys. 2004, 121, 902-913.

(33) Detoni, S.; Hadži, D. Hydroxyl Bands in the Infra-Red Spectra of Organophosphoric and Phosphinic Acids. Spectrochim. Acta 1964, 20, 949-955.

(34) Peppard, D. F.; Ferraro, J. R.; Mason, G. W. The Preparation, Physical Properties and Infra-Red Spectra of Several New Organophosphonates. J. Inorg. Nucl. Chem. 1959, 12, 60-70.

(35) Milo, A.; Neel, A. J.; Toste, F. D.; Sigman, M. S. A DataIntensive Approach to Mechanistic Elucidation Applied to Chiral Anion Catalysis. Science 2015, 347, 737-743.

(36) Milo, A.; Bess, E. N.; Sigman, M. S. Interrogating Selectivity in Catalysis Using Molecular Vibrations. Nature 2014, 507, 210-214.

(37) Hadži, D. Spectroscopic and Structural Aspects of Very Strong Hydrogen Bonds. Chimia 1972, 26, 7-13.

(38) Dreyer, J. Hydrogen-Bonded Acetic Acid Dimers: Anharmonic Coupling and Linear Infrared Spectra Studied with DensityFunctional Theory. J. Chem. Phys. 2005, 122, 184306.

(39) Ultrafast Hydrogen Bonding Dynamics and Proton Transfer Prosesses in the Condensed Phase; Elsaesser, T., van der Akker, H., Eds.; Springer Netherlands: Dordrecht, 2002.

(40) Bratos, S. Profiles of Hydrogen Stretching IR Bands of Molecules with Hydrogen Bonds: A Stochastic Theory. I. Weak and Medium Strength Hydrogen Bonds. J. Chem. Phys. 1975, 63, 34993509.

(41) Šoptrajanov, B.; Jovanovski, G.; Kuzmanovski, I.; Stefov, V. Fourier Transform Vibrational Spectra of Magnesium Hydrogenphosphate Trihydrate. I. The O-H Stretching Region. Spectrosc. Lett. 1998, 31, 1191-1205.

(42) Evans, J. C. Further Studies of Unusual Effects in the Infrared Spectra of Certain Molecules. Spectrochim. Acta 1960, 16, 994-1000.

(43) Stingel, A. M.; Petersen, P. B. Interpreting Quasi-Thermal Effects in Ultrafast Spectroscopy of Hydrogen-Bonded Systems. J. Phys. Chem. A 2018, 122, 2670-2676.

(44) Giebels, I. A. M. E.; van den Broek, M. A. F. H.; Kropman, M. F.; Bakker, H. J. Vibrational Dynamics of Hydrogen-Bonded HClDiethyl Ether Complexes. J. Chem. Phys. 2000, 112, 5127-5132.

(45) Daly, C. A.; Streacker, L. M.; Sun, Y.; Pattenaude, S. R.; Hassanali, A. A.; Petersen, P. B.; Corcelli, S. A.; Ben-Amotz, D. Decomposition of the Experimental Raman and Infrared Spectra of Acidic Water into Proton, Special Pair, and Counterion Contributions. J. Phys. Chem. Lett. 2017, 8, 5246-5252.

(46) Cannon, C. G. The Nature of Hydrogen Bonding. Spectrochim. Acta 1958, 10, 341-368.

(47) Fournier, J. A.; Carpenter, W. B.; Lewis, N. H. C.; Tokmakoff, A. Broadband 2D IR Spectroscopy Reveals Dominant Asymmetric $\mathrm{H}_{5} \mathrm{O}_{2}{ }^{+}$Proton Hydration Structures in Acid Solutions. Nat. Chem. 2018, 10, 932-937.

(48) Rueping, M.; Azap, C.; Sugiono, E.; Theissmann, T. Brønsted Acid Catalysis: Organocatalytic Hydrogenation of Imines. Synlett 2005, 15, 2367-2369.

(49) Rueping, M.; Sugiono, E.; Azap, C. A Highly Enantioselective Brønsted Acid Catalyst for the Strecker Reaction. Angew. Chem., Int. Ed. 2006, 45, 2617-2619.

(50) Hamm, P.; Zanni, M. Concepts and Methods of 2D Infrared Spectroscopy; Cambridge University Press: Oxford, U.K., 2011. 
(51) Elsaesser, T. Two-Dimensional Infrared Spectroscopy of Intermolecular Hydrogen Bonds in the Condensed Phase. Acc. Chem. Res. 2009, 42, 1220-1228.

(52) Bredenbeck, J.; Helbing, J.; Kolano, C.; Hamm, P. Ultrafast 2DIR Spectroscopy of Transient Species. ChemPhysChem 2007, 8, 1747-1756.

(53) Cho, M. Coherent Two-Dimensional Optical Spectroscopy. Chem. Rev. 2008, 108, 1331-1418.

(54) Cyran, J. D.; Nite, J. M.; Krummel, A. T. Characterizing Anharmonic Vibrational Modes of Quinones with Two-Dimensional Infrared Spectroscopy. J. Phys. Chem. B 2015, 119, 8917-8925.

(55) Mazur, K.; Bonn, M.; Hunger, J. Hydrogen Bond Dynamics in Primary Alcohols: A Femtosecond Infrared Study. J. Phys. Chem. B 2015, 119, 1558-1566.

(56) Marekha, B. A.; Hunger, J. Hydrophobic Pattern of Alkylated Ureas Markedly Affects Water Rotation and Hydrogen Bond Dynamics in Aqueous Solution. Phys. Chem. Chem. Phys. 2019, 21, 20672-20677.

(57) Shim, S.-H.; Zanni, M. T. How to Turn Your Pump-Probe Instrument into a Multidimensional Spectrometer: 2D IR and Vis Spectroscopies via Pulse Shaping. Phys. Chem. Chem. Phys. 2009, 11, $748-761$.

(58) Hillegas, C. W.; Tull, J. X.; Goswami, D.; Strickland, D.; Warren, W. S. Femtosecond Laser Pulse Shaping by Use of Microsecond Radio-Frequency Pulses. Opt. Lett. 1994, 19, 737-739.

(59) Shim, S.-H.; Strasfeld, D. B.; Fulmer, E. C.; Zanni, M. T. Femtosecond Pulse Shaping Directly in the Mid-IR Using AcoustoOptic Modulation. Opt. Lett. 2006, 31, 838-840.

(60) Shim, S.-H.; Strasfeld, D. B.; Ling, Y. L.; Zanni, M. T. Automated 2D IR Spectroscopy Using a Mid-IR Pulse Shaper and Application of this Technology to the Human Islet Amyloid Polypeptide. Proc. Natl. Acad. Sci. U. S. A. 2007, 104, 14197-14202.

(61) Middleton, C. T.; Woys, A. M.; Mukherjee, S. S.; Zanni, M. T. Residue-Specific Structural Kinetics of Proteins through the Union of Isotope Labeling, Mid-IR Pulse Shaping, and Coherent 2D IR Spectroscopy. Methods 2010, 52, 12-22.

(62) Strasfeld, D. B.; Shim, S. H.; Zanni, M. T. Controlling Vibrational Excitation with Shaped Mid-IR Pulses. Phys. Rev. Lett. 2007, 99, 1-4.

(63) Strasfeld, D. B.; Middleton, C. T.; Zanni, M. T. Mode Selectivity with Polarization Shaping in the Mid-IR. New J. Phys. 2009, $11,105046$.

(64) Rekik, N.; Ghalla, H.; Hanna, G. Explaining the Structure of the OH Stretching Band in the IR Spectra of Strongly Hydrogen-Bonded Dimers of Phosphinic Acid and Their Deuterated Analogs in the Gas Phase: A Computational Study. J. Phys. Chem. A 2012, 116, 44954509.

(65) Van Hoozen, B. L.; Petersen, P. B. Origin of the Hadži ABC Structure: An Ab Initio Study. J. Chem. Phys. 2015, 143, 184305.

(66) Parr, R. G.; Yang, W. Density-Functional Theory of the Electronic Structure of Molecules. Annu. Rev. Phys. Chem. 1995, 46, $701-728$.

(67) Kohn, W.; Sham, L. J. Self-Consistent Equations Including Exchange and Correlation Effects. Phys. Rev. 1965, 140, A1133A1138.

(68) Hohenberg, P.; Kohn, W. Inhomogeneous Electron Gas. Phys. Rev. 1964, 136, B864-B871.

(69) Becke, A. D. Density-Functional Thermochemistry. III. The Role of Exact Exchange. J. Chem. Phys. 1993, 98, 5648-5652.

(70) Petersson, G. A.; Bennett, A.; Tensfeldt, T. G.; Al-Laham, M. A.; Shirley, W. A.; Mantzaris, J. A Complete Basis Set Model Chemistry. I. The Total Energies of Closed-shell Atoms and Hydrides of the First-row Elements. J. Chem. Phys. 1988, 89, 2193-2218.

(71) Petersson, G. A.; Al-Laham, M. A. A Complete Basis Set Model Chemistry. II. Open-shell Systems and the Total Energies of the Firstrow Atoms. J. Chem. Phys. 1991, 94, 6081-6090.

(72) Grimme, S.; Ehrlich, S.; Goerigk, L. Effect of the Damping Function in Dispersion Corrected Density Functional Theory. J. Comput. Chem. 2011, 32, 1456-1465.
(73) Scalmani, G.; Frisch, M. J. Continuous Surface Charge Polarizable Continuum Models of Solvation. I. General Formalism. J. Chem. Phys. 2010, 132, 114110.

(74) Frisch, M. J.; Trucks, G. W.; Schlegel, H. B.; Scuseria, G. E.; Robb, M. A.; Cheeseman, J. R.; Scalmani, G.; Barone, V.; Petersson, G. A.; Nakatsuji, H.; et al.Gaussian 16, Revision B.01; Gaussian, Inc.: Wallingford, CT, 2016.

(75) Colbert, D. T.; Miller, W. H. A Novel Discrete Variable Representation for Quantum Mechanical Reactive Scattering via the S-Matrix Kohn Method. J. Chem. Phys. 1992, 96, 1982-1991.

(76) Hanna, G.; Geva, E. Computational Study of the Signature of Hydrogen-Bond Strength on the Infrared Spectra of a HydrogenBonded Complex Dissolved in a Polar Liquid. Chem. Phys. 2010, 370, 201-207.

(77) Bratos, S.; Ratajczak, H. Profiles of Hydrogen Stretching IR Bands of Molecules with Hydrogen Bonds: A Stochastic Theory. II. Strong Hydrogen Bonds. J. Chem. Phys. 1982, 76, 77-85.

(78) Rosenfeld, D. E.; Kwak, K.; Gengeliczki, Z.; Fayer, M. D. Hydrogen Bond Migration between Molecular Sites Observed with Ultrafast 2D IR Chemical Exchange Spectroscopy. J. Phys. Chem. B 2010, 114, 2383-2389.

(79) Hunger, J.; Stoppa, A.; Thoman, A.; Walther, M.; Buchner, R. Broadband Dielectric Response of Dichloromethane. Chem. Phys. Lett. 2009, 471, 85-91.

(80) Nibbering, E. T. J.; Dreyer, J.; Oliver, K.; Bredenbeck, J.; Hamm, P.; Elsaesser, T. Vibrational Dynamics of Hydrogen Bonds. Anal. Control Ultrafast Photoinduced React. 2007, 87, 619-687.

(81) Lindemann, R.; Zundel, G. Polarizability, Proton Transfer and Symmetry of Energy Surfaces of Carboxylic Acid-N-Base Hydrogen Bonds. Infrared Investigations. J. Chem. Soc., Faraday Trans. 2 1977, $73,788-803$.

(82) Balasubramanian, M.; Reynolds, A.; Blair, T. J.; Khalil, M. Probing Ultrafast Vibrational Dynamics of Intramolecular Hydrogen Bonds with Broadband Infrared Pump-Probe Spectroscopy. Chem. Phys. 2019, 519, 38-44.

(83) Hunger, J.; Sonnleitner, T.; Liu, L.; Buchner, R.; Bonn, M.; Bakker, H. J. Hydrogen-Bond Dynamics in a Protic Ionic Liquid: Evidence of Large-Angle Jumps. J. Phys. Chem. Lett. 2012, 3, 30343038.

(84) Zheng, Z.-P.; Fan, W.; Roy, S.; Mazur, K.; Nazet, A.; Buchner, R.; Bonn, M.; Hunger, J. Ionic Liquids: Not Only Structurally but Also Dynamically Heterogeneous. Angew. Chem., Int. Ed. 2015, 54, 687-690.

(85) Rezus, Y. L. A.; Bakker, H. J. On the Orientational Relaxation of HDO in Liquid Water. J. Chem. Phys. 2005, 123, 114502.

(86) Mitra, R.; Niemeyer, J. Dual Brønsted-Acid Organocatalysis: Cooperative Asymmetric Catalysis with Combined Phosphoric and Carboxylic Acids. ChemCatChem 2018, 10, 1221-1234. 